\title{
Designing and engineering through collaboration and negotiation
}

\author{
N.P. Suh \\ The Park Centre for Complex Systems, \\ Massachusetts Institute of Technology, \\ Cambridge, MA 02139, USA \\ E-mail: npsuh@mit.edu
}

\begin{abstract}
The development of complex engineering systems requires collaboration and negotiation. It is to make correct decisions based on customer needs, Functional Requirements, Design Parameters, and Process Variables. There are two basic elements: the system and the process used for collaboration and negotiation. The system is to assure that the project has the best information and knowledge. Systems and processes are needed to minimise the cost of development, to execute the project on schedule, and to deliver a highly robust, efficient and reliable product. This paper proposes heuristic rules for collaboration and negotiation based on axiomatic design theory and complexity theory.
\end{abstract}

Keywords: collaboration; negotiation; engineering systems; axiomatic design; complexity.

Reference to this paper should be made as follows: Suh, N.P. (2009) 'Designing and engineering through collaboration and negotiation', Int. J. Collaborative Engineering, Vol. 1, Nos. 1/2, pp.19-37.

Biographical notes: N.P. Suh is the President of Korea Advanced Institute of Science and Technology (KAIST) since July 2006. Previously, he had been at MIT since 1970, where he was the Ralph E. and Eloise F. Cross Professor and the Head of the Department of Mechanical Engineering. In 1984-1988, he was at the US National Science Foundation where he was in charge of engineering. His fields of interest include design, manufacturing, tribology, and materials processing. He is the author of over 300 papers and seven books, holds more than 60 patents, and edited several books. He received seven honourary degrees.

\section{Introduction}

Innovation is the engine for economic growth. Semiconductors have enabled the development of modern telecommunications, air transportation, manufacturing of precision parts and equipment, and many others. Information technology has changed nearly all aspects of societal and personal functions, behaviour, and operations. The advances in molecular biology have been equally far-reaching, changing drug development, healthcare, agriculture, and pharmacology. There are many other examples that show the impact of technology on economy and society. 
The ability of organisations to innovate depends on how well the people within the organisation collaborate and/or negotiate. ${ }^{1}$ Therefore, many industrial companies have instituted organised systems and processes to promote effective collaboration and negotiation, albeit with mixed results.

Innovations in many fields share several common elements: nucleation of innovative ideas, product design and development, entrepreneurial systems for conversion of ideas to products, manufacturing, marketing, procurement, etc. Most of these activities require human collaboration. Notwithstanding many of the revolutionary changes brought about by advances in science and technology, human collaboration and interaction is not yet a fully developed intellectual discipline and thus may constitute an impediment to the innovation process.

There are two different kinds of engineering and development projects:

- creation of completely new systems and/or products (e.g., the Space Shuttle)

- incremental advances and modification of existing products (e.g., conventional passenger cars).

The development of these complicated products often involves large teams of designers, engineers, and managers. Depending on how the project is organised and managed, the outcome of the collaboration and negotiation will be different. The quality of collaboration can determine the functionality, reliability, and robustness of the product as well as the lifecycle cost. The fundamental reason for the different outcomes is that the design process is not a 'one-to-many' mapping process (i.e., in design, the uniqueness theorem does not apply because for every Functional Requirement (FR), there are many possible solutions) and that collaborators can make wrong decisions.

Engineering development projects are organised either along a functional group (e.g., control engineering) or physical group (e.g., power steering, air conditioners). Some companies have a matrix organisation to capture the good features of both functional and physical organisations. These different organisational structures affect the effectiveness of collaboration among the participants and thus the productivity of the organisation. When projects are organised without the benefit of a theoretical framework, the effectiveness and the productivity of the organisation may be compromised. This lack of organising principles for collaboration may be one of the reasons why the structure of technical organisations is frequently changed hoping to address shortcomings of their system and process for collaboration and negotiation.

For system-development projects to succeed, collaboration must also exist between technologists and non-technologists (Sohlenius, 2005). For example, a close collaboration between technologists and marketing groups is required to overcome both technical risks and marketing risks in transitioning a technological success into commercial success. This is not a trivial task (Branscomb and Auerswald, 2001).

When collaborative work is not properly managed, the time and effort required to execute an engineering project may increase nonlinearly with the number of participants. Once the concept for a product is selected by a company for production, a large number of engineers and designers are likely to be assigned to the project. The number of engineers, designers and managers required will vary as a function of the nature and the scope of the project. When many people work in a team, they must strive to achieve the common goal of the project. The work must be properly sub-divided, relevant data and 
facts should not be overlooked, all the contingencies that the project must deal with should be considered, and the workload must be reasonable for the given level of staffing.

In a symphony orchestra, all of the players have musical notes that guide them, and the conductor coordinates and directs the musicians to play the symphony in harmony. Engineering collaboration should be similar to a symphony orchestra. Unfortunately, in engineering, the players (i.e., engineers) often try to collaborate to create the music without the sheet music, and the conductor (i.e., the chief engineer) tries to compose the music without a set of rules, or musical theory.

The purpose of this paper is an attempt to outline how engineering collaboration can be made to be like symphony orchestra.

\section{Basic steps in collaboration and negotiation}

Collaboration in a project can quickly become chaotic with an increase in the number of people, especially when they do not understand the project's ultimate goal, and are not given the general framework for collaboration.

An ideal arrangement for collaboration should consist of the following general steps:

- all the participants should understand the overall goal of the project (i.e., 'what' the collaboration must achieve)

- the strategy for achieving the goal of the project should be designed and understood by key participants

- a well-established process should guide the mode of collaboration among all participants (e.g., criteria for good decisions, system hierarchy, and information flow).

These steps will be discussed based on the axiomatic design theory.

\section{Basic steps in engineering design}

When a large system is to be designed, there are many issues the designers of engineering systems must deal with and ultimately agree on, such as:

- Who are our customers?

- What does the customer need?

- Of the many possible sets of FRs that can be chosen to meet the customer needs, which FRs should be selected?

- What are the best means of satisfying the FRs?

- What should the design be like to make the product robust and reliable?

- How do we manufacture the products in the most robust, economical and reliable way?

- How do we test the product to determine its performance? 
- How do we verify the correctness of our decisions?

Unless the design is done and executed by only one person, each issue identified above may require the involvement of many, including those from engineering, marketing, and manufacturing. The final decisions should be made by the system architect after considering the inputs from all stakeholders.

Many decisions must be made to deal with the questions raised above. But how do we know that the decisions are correct or acceptable? Some decisions, regardless who made them, and how many participants were involved in arriving at the decision, may be incorrect or involve the risk of creating unintended results. Basic principles that provide metrics for correctness (or acceptability) must be established, to be sure that correct decisions are made. Are there basic principles that can guide collaborative efforts? For example, NASA adopted the use of the polyurethane foam on the outside surface of the external tank (that contains liquid hydrogen and oxygen) of the Space Shuttle as a means of preventing ice formation on the tank surface. Notwithstanding extensive trade studies conducted in aerospace industries, this design decision caused the failure of one of the Space Shuttle missions because a loose foam piece struck and damaged the wing of the Orbitor. This 'wrong' decision, which was caused by the coupling of one FR with another FR, cost lives of many brave people, incurred billions of dollars to continue the operation, and set back the progress of the manned space program by a couple of decades. This example illustrates that collaboration involving a large number of people does not necessarily lead to 'correct' decisions in the absence of rational decision-making criteria to supplement the trade study.

\section{System and process for collaboration and negotiation}

Effective collaboration and negotiation needs systems and processes that govern group interactions. The purpose of the system is to organise projects and participants so as to maximise their effectiveness and achieve the goals of the project. The process outlines an algorithm for collaboration and negotiation to reinforce the strengths of all participants, minimise confusion, and deal with the resulting disagreement. Sometimes well-meaning people can disagree because of their misunderstanding of the issues involved, and the lack of fundamental framework for good decision-making. Projects must be organised with a clear idea on how their sub-tasks are related (i.e., uncoupled, decoupled, or coupled). Otherwise, collaboration and negotiation often become chaotic. A system that clearly shows the dependencies among sub-tasks can greatly facilitate communication and coordination and hence improve collaboration and negotiation.

In an ideal situation, both the system and the process for collaboration and negotiation must be designed based on basic principles. To achieve this goal, the system and the process for collaboration and negotiation must be based on design principles that define acceptable designs and provides an overall framework.

The existence of systems for collaboration and negotiation may not make it 'easy', because the system does not provide the contents that go into it to arrive at a right set of goals and solutions. A system is a framework that facilitates making good decisions. The intellectual content that goes into the systematic framework is discipline specific, and thus requires experts of the subject matter. 
The system for collaboration may assume many different forms. In this paper, it will be assumed that the system will follow the product-development hierarchy established by the axiomatic design theory. The information flow will be assumed to follow the hierarchical system architecture established by the axiomatic design.

The process of collaboration in engineering system development will be assumed to be governed by a set of rules, which are presented later after the basic framework of axiomatic design and complexity theories is presented. The process may also be governed by organisational protocols, which will not be discussed in this paper.

\section{When and how to collaborate and negotiate: systems aspect}

The need to collaborate and negotiate is determined by two things: complexity of the development project and the financial and other resource commitments required to execute the project. These two conditions are present when the project size is large, in terms of the number of people and FRS that must be satisfied. In executing such projects, a systematic approach such as the one described below is required.

Product design and development process may be described in terms of the four domains of axiomatic design shown in Figure 1 (Suh, 1990, 2001). The first domain is the customer domain, in which the attributes and needs of the customers are characterised as Customer Attributes (CAs). The second domain is the functional domain, in which the customer needs described in the first domain are translated into a set of FRs. The third domain is the physical domain, which is characterised by a set of Design Parameters (DPs) chosen to satisfy the FRs. The last domain is the process domain, which consists of a set of Process Variables (PVs) that can create the DPs of the third domain. The characteristic vectors, $\{F R s\}$ of the functional domain, $\{$ DPs $\}$ of the physical domain, and $\{\mathrm{PVs}\}$ of the process domain are decomposed to the leaf-level FRs, DPs, and PVs to produce a product that satisfies the highest-level FRs. In Suh (1990, 2001), many examples are given that illustrate the basic concept.

Figure 1 Four domains of the design world

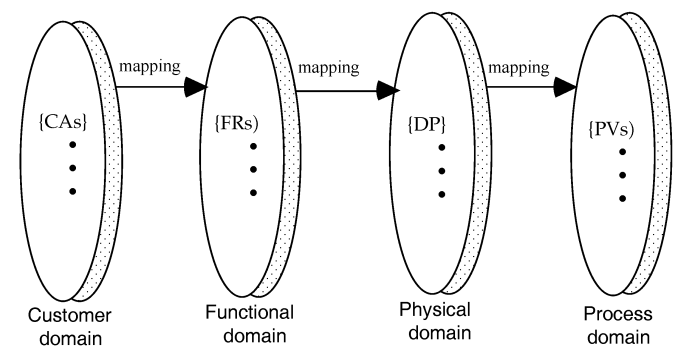

The higher-level FRs and DPs must be decomposed to lower and lower levels until all the design details are addressed. The decomposition is achieved by zigzagging between the functional and the physical domain. Since there may be many DPs that can satisfy a given FR, it is important that the best DP be chosen to produce the most robust design. Throughout the decomposition process, collaboration and negotiation may be needed to accelerate the design process.

It should be noted that the concept of four domains has been applied to a variety of different problems, including design of machines, hardware, software, organisations, 
manufacturing systems, materials, and strategies (Suh, 2001, 2005). In the following discussions, product design will be used to illustrate the basic concept.

\subsection{Collaboration in determining the customer needs}

In the customer domain, we must be able to identify the customer needs accurately since the subsequent design process is directly affected and controlled by the customer needs. The process used to define customer needs is different from project to project, company to company, and product to product. Therefore, the need to collaborate and negotiate in defining the customer needs also differs. For example, in 1959 a small start-up plastics company that made simple plastic articles (such as dishes for ice cream and cups for soft drinks) came up with a new patented process that has become commercially important. In this case, the customer's needs were identified by the company's president and owner. He identified a weakness of a product - a foamed cup made of expanded polystyrene - made by a competitor, which was well protected by patents. He found that the wall thickness of the competitor's foamed cup made of expandable polystyrene beads was too thick to be used in vending machines, because the stacking height of the cups in a vending machine is determined by the nesting height of the cups, which is strongly affected by the wall thickness of the cup, although its thermal insulation property was excellent in handling hot coffee. He decided that there was a need for a thin-walled cup but with good thermal insulation that can be used in vending machines. He assigned a young engineer to design and develop such an alternative cup. Such a product and process was developed during the next six months, which was patented, and is used to this day in making dishes, cups, and trays. This is a case where successful technological innovation was made with limited collaboration and negotiations.

An extreme opposite example is the manned space program of the National Aeronautics and Space Agency (NASA). NASA has spent many years trying to determine what attributes their customers will support in terms of manned space flight. Their ultimate customers are the taxpayers, but the immediate customers who will make the funding decision are the Congress, the President, and the Office of Management and Budget of the US government. NASA has spent a large sum (perhaps more than hundreds of millions of dollars) to develop manned space systems that can replace the Space Shuttle program, which can be sold to Congress, the White House, and to the public.

In many companies, however, the project size is much smaller than the NASA's manned space program but much larger than the plastic cup project. For example, the development of equipment required in semiconductor manufacturing (except optical lithography which is an order of magnitude more complicated) may involve 20-60 engineers and a budget of $\$ 30-\$ 100$ million. A significant part of this cost is due to the ad hoc nature - the "design/build/test/fix" cycle - of the product development process used in some companies, which escalates the cost. Fortunately the customer needs in semiconductor industry are relatively better known by the road map published by the semiconductor industry.

In conducting product development, a great deal of data collection, collaboration, and negotiation are required. Initially the size of the team is relatively small, but it grows with the development of the project. Identifying customer needs is a difficult task unless the company has a dominant position in the market and thus can have better access to the key customers, and has influence in establishing the customer needs. 
To determine customer needs, companies survey customer preference. Such a market study can be subjective and may reflect the bias of the surveyors. Researchers in economics and operations research have investigated the determination of customer preference. A well-known work is the Arrow's impossibility theorem (Arrow, 1951). It states that the group preference determined cannot be determined from a collection of individual preferences given by discrete ranking. However, the conclusion of this theorem was shown to be invalid when the individual preference is evaluated in terms of cardinal utility function that are based on a ratings of continuous alternatives (Harsanyi, 1955; Keeney, 1976), where utility is a measure of the happiness or satisfaction gained consuming good and services, and where cardinal utility is defined as a measurable quantity that could be aggregated across individuals and measures the preference of an individual towards a certain commodity. If cardinal utility function is defined in a 'continuous' option space provided by physics based design, then the Arrow's criteria of rationale democratic decision making are not violated and therefore, the collaborative effort can lead to correct decisions ( $\mathrm{Lu}, 2006 \mathrm{a})$.

\subsection{Collaboration and negotiation in determining FRs, DPs, and constraints}

Once the customer's needs are determined, the task of the designers is to establish a set of FRs, which will satisfy customer needs. The functionality is defined as a minimum set of independent requirements that completely characterise the functions of a product (Suh, 1990). The establishment of the FRs is an important step in designing engineering systems, because the ultimate performance of the product is determined by the established FRs. FRs must be satisfied within the bounds imposed on the solution by constraints.

In some cases, one person can establish all the FRs without the need for collaboration and negotiation. However, in many cases, FRs are determined by a large number of people with diverse backgrounds. When a group of participants establish the FRs, collaboration and negotiation are an essential part of the group activity. Group decisions are preferable when the task is too involved for one person to possess and know all the FRs that a product must satisfy. Unfortunately, when a large number of people participate in determining FRs, the decision-making time increases with the number of participants, unless the collaborators are given basic principles for making design decisions.

Since the FRs thus established is independent from each other, the design process must choose specific DPs that do not couple the FRs. This requirement is formally stated as the Independence Axiom ("Maintain the independence of FRs") in axiomatic design theory. An acceptable design that satisfies the Independence Axiom is an uncoupled design or a decoupled design. In an ideal design the number of FRs and the number of DPs are the same (which is Theorem 4 of Axiomatic Design Theory). The design matrix that relates FRs to DPs is a diagonal matrix for an uncoupled design and a triangular design for a decoupled design. When the design matrix is not diagonal or triangular, the design is a coupled design, which is not an acceptable design because of the coupling of FRs. Functional independence, the process of developing uncoupled or decoupled design, and the concept of design matrix are discussed and analysed in Suh $(1990,2001)$.

The highest-level FRs and DPs may not be implemented because of the lack of design details. In this case, FRs and DPs must be decomposed. To decompose FR and DP characteristic vectors, we must zigzag between the domains. That is, we start out in the 
'what' domain (i.e., the functional domain) and go to the 'how' domain (i.e., the physical domain). This is illustrated in Figure 2. From an FR in the functional domain, we go to the physical domain to conceptualise a design and determine its corresponding DP. Then, we come back to the functional domain to create FR1 and FR2 at the next level that collectively satisfies the highest-level FR. FR1 and FR2 are the FRs for the highest level DP. Then we go to the physical domain to find DP1 and DP2 (children of the highest-level FR and DP) by conceptualising a design at this level, which satisfy FR1 and FR2, respectively. This process of decomposition is pursued until the FR can be satisfied without further decomposition when all of the branches reach the final state. The final state is indicated by thick boxes in Figure 2, which are called the 'leaf' or 'leaves'.

Collaborators in engineering projects must understand the design process and the design hierarchy to be able to find the right partners for collaboration and to seek information from the right people who are working on the right FRs and DPs as represented by the design hierarchy.

Figure 2 Zigzagging to decompose FRs and DPs in the functional and the physical domains and to create the FR and DP hierarchies. Boxes with thick lines represent 'leaves' that do not require further decomposition

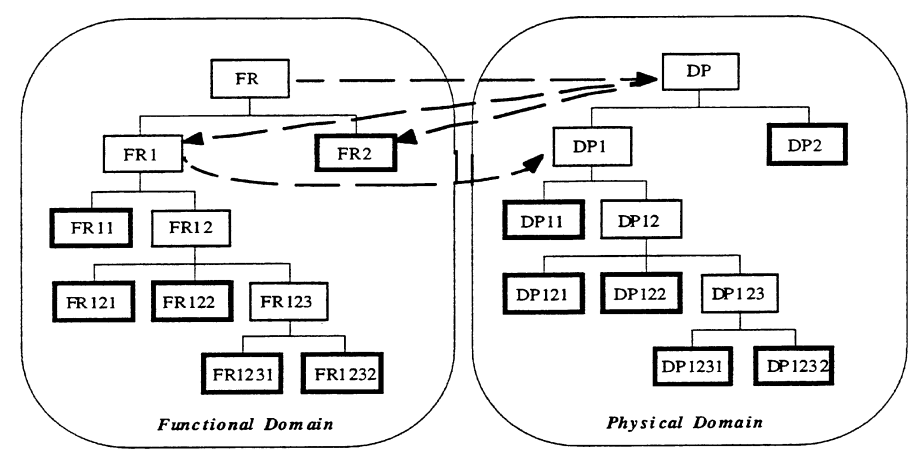

To be sure that we have made the right design decision, we must write down the design equation $-\{\mathrm{FRs}\}=[\mathrm{A}]\{\mathrm{DPs}\}-$ at each level of decomposition. For example, in the case shown in Figure 2, after FR and DP are decomposed into FR1, FR2, DP1 and DP2, we must write down the design equation to indicate our design intent at this level. Since we know that the design must be either uncoupled or decoupled designs, the intended design must have either a diagonal or a triangular matrix. All subsequent lower-level design decisions must be consistent with this high-level design decision. Design Matrix [A] can be used as a governing matrix for group collaboration and negotiation.

At each level of decomposition, the design decisions made must be consistent with all higher-level design decisions that were already made. That is, if the highest-level design matrix is a diagonal matrix, all lower-level decisions must not make - either intentionally or inadvertently - the off-diagonal elements of the highest-level design matrix non-zeroes. To check this fidelity and consistency of design decisions, the full design matrix must be constructed by combining all lower-level design matrices into a single master matrix.

To decompose FRs and DPs, the designer must zigzag. We cannot decompose the highest-level FRs unless we first conceptualise DPs that can satisfy these highest-level FRs. Therefore, when we define the FRs in a solution-neutral environment, we have to 
'zig' to the physical domain, and after proper DPs are chosen, we have 'zag' to the functional domain for further decomposition. Details are given in Suh (1990, 2001).

In designing a manufacturing system to make the chosen DPs, a mapping similar to the FR/DP mapping must be done. To have an acceptable design, both the FR/DP design matrix and the DP/PV design matrix must be diagonal or triangular. The details of the mapping and decomposition process are discussed in Suh $(1990,2001)$. In this DP to PV mapping process, the collaboration and negotiation between the designer and the manufacturing engineer is essential to insure that products that can easily be manufactured are designed.

When collaborators in an engineering system development project understand the design process of axiomatic design, they will know what constitutes good decisions in design. They will also be able to seek and convey the right information to other participants based on the design hierarchy. They will know how the information should flow and how collaborators must interact to make the right decision based on the design hierarchy. Without this kind of system, it will be difficult to make decisions by a group of people, who may have different educational and experiential backgrounds.

\section{Collaboration and information content}

Collaboration and negotiation will be more effective when all the participants understand how information content is defined in axiomatic design and how the Information Axiom applies.

The second axiom of the axiomatic design theory, the Information Axiom, states that the information content must be minimised, which means that the final product performance must be within the specified allowable variation for each FR. The information content is zero when the system performance (given by the system range) is always inside the allowable variation range (given by the design range). When the system range is outside of the design range the ratio of the overlap between the design range and the system range determines the information content (see Figure 3 ). In negotiation sciences, the concepts of Better Alternatives To Negotiated Agreement (BATNA) and 'reserved value' are critical. Both concepts are related to the information content in Axiomatic Design (Lu, 2006b).

The information content $I$ of a system with $\mathrm{n}$ FRs is defined as

$$
I_{\text {sys }}=-\log _{2} P_{\{n\}}
$$

where $P_{\{n\}}$ is the joint probability that all $n$ FRs are satisfied. When the FRs are independently satisfied, equation (1) may be written as

$$
I=-\sum_{i=1}^{n} \log _{2} P_{i}
$$

where $P_{i}$ is the probability of the system range of $\mathrm{FR}_{i}$ being inside the design range for the FR. 
The important implication of the Information Axiom for collaboration and negotiation is that collaborators must seek solutions that will minimise the information content, and eliminate the waste and inefficiency brought about by 'information overload'.

Figure 3 The goal of design decision is to make the system range to be inside the design range. Information content is measured by the ratio of areas of the common range to the system range

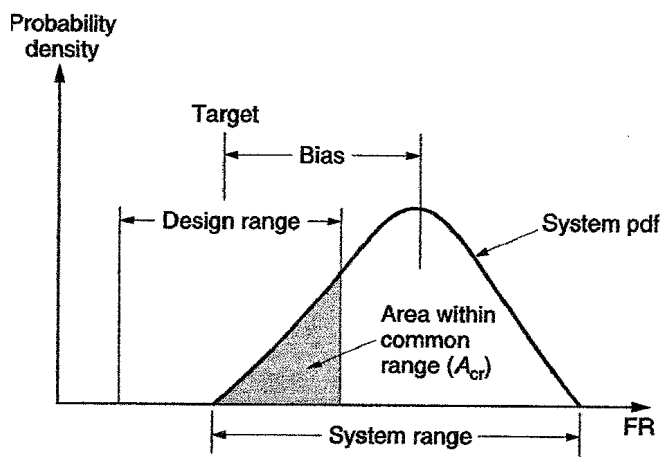

\section{Complexity in collaboration and negotiation}

When the overlap between the system range and the design range is small, it is difficult to satisfy the FRs and therefore, the task of achieving the FRs appears to be more complex. In other words, the certainty of always achieving the task defined by an FR is governed by the relationship between the design range and the system range of a given FR. Using this observation as the starting point, a complexity theory was advanced, where the complexity is defined as "a measure of uncertainty in satisfying the FRs" (Suh, 2005).

The role of collaboration and negotiation may also be better understood in terms of complexity theory, which extends the notion of information content defined in the axiomatic design theory.

Based on this definition of complexity, we can show that there are four different kinds of complexities, all of which have definite implications to collaboration and negotiation in developing engineering systems. The purpose of collaboration and negotiation should be to eliminate or reduce complexity by examining the four different kinds of complexity.

\subsection{Four different complexities}

Complexity can be a function of time or can be completely independent of time, depending on whether or not the system range shown in Figure 2 changes as a function of time. Therefore, complexity can be classified into the following two kinds: time-dependent complexity and time-independent complexity.

Time-independent complexity can be further classified into two different types: time-independent real complexity and time-independent imaginary complexity. 


\subsubsection{Real complexity}

As the terms implies, real complexity is a result of not satisfying the FR with $100 \%$ certainty. Figure 3 show such a case. That is, the real complexity is a consequence of the system range not being inside the design range. Therefore, the real complexity measures the same thing as the information content of the axiomatic design theory.

\subsubsection{Imaginary complexity}

The imaginary complexity may exist if the decoupled design has more than one FR. When the design is a decoupled design characterised by a triangular design matrix, a complexity called the imaginary complexity can exist. In this case, if the DPs of a decoupled design are varied randomly without following the order specified by the triangular design matrix, the system appears to be very complex, which is defined as the imaginary complexity. Consider a combinatorial lock. Although it is simple to open it if we know the numbers and the sequence, it is very complex to open it in the absence of this information. That is, a well-designed decoupled system may appear to be complex if we do not understand the system in terms of the design matrix. A system with imaginary complexity can satisfy the FRs at all times if we vary DPs in the right order, but when we lack the knowledge on the system design, we may be unable to make the system satisfy the FRs.

Consider a decoupled design with $n$ FRs and $n$ DPs given by the triangular matrix:

$$
\left\{\begin{array}{l}
\mathrm{FR}_{1} \\
\mathrm{FR}_{2} \\
\cdots \\
\cdots \\
\mathrm{FR}_{n}
\end{array}\right\}=\left[\begin{array}{lllll}
\mathrm{X} & 0 & \ldots & \ldots & 0 \\
\mathrm{X} & \mathrm{X} & \ldots & \ldots & 0 \\
\ldots & \ldots & \ldots & \ldots & 0 \\
\ldots & \ldots & \ldots & \ldots & 0 \\
\mathrm{XX} & \ldots & \ldots & \mathrm{X}
\end{array}\right]\left\{\begin{array}{l}
\mathrm{DP}_{1} \\
\mathrm{DP}_{2} \\
\cdots \\
\cdots \\
\mathrm{DP}_{n}
\end{array}\right\} .
$$

The design represented by equation (3) satisfies the Independence Axiom if we change the DPs in the order given. Thus it can be implemented because there is no uncertainty associated with it if the DPs are changed in the order indicated in equation (3) and if each of the system ranges is inside its associated design range. If the system range is inside the design range for all $\mathrm{FR}_{\mathrm{i}}$, then the real complexity is equal to zero. However, the decoupled design given by equation (3) can be a source of imaginary complexity, despite the fact that the design does satisfy the Independence Axiom and the real complexity is zero. This imaginary uncertainty exists only in the mind of the designer when the designer does not explicitly design as represented by equation (3).

Suppose that even the designer does not recognise a design is decoupled and thus does not know that the DPs must be changed in a proper order to make the design achieve the given set of $n$ FRs. If the designer resorts to trial-and-error methods of evaluation, trying many different sequences of DPs to satisfy the FRs, there is $n$ ! sequences of DPs, of which only one is correct. Then the probability of finding the right sequence of $n$ DPs to satisfy the entire set of $n$ FRs is given by

$$
P=\frac{1}{n !} \text {. }
$$


For example, when $n$ is 5 , the probability of finding the right sequence is 0.008 , which is quite low. Therefore, this design appears to be very complex because the uncertainty is large. However, this uncertainty is artificially created by lack of understanding of the system designed and the situation will become even more complex when a group of engineers is involved. Therefore, this kind of uncertainty is defined as imaginary uncertainty. If we denote imaginary complexity as $C_{I}$, then the maximum imaginary complexity may be related to the probability of finding the right sequence given by equation (3) as

$$
\left(C_{I}\right)_{\max }=-\log n !
$$

In collaboration and negotiation, a poorly designed design process is likely to lead to unproductive and nearly endless discussion, if the participants do not understand that they are arguing about an imaginary complexity problem. For example, in discussion of the NASA's mission to Mars, we should not discuss the mission and the equipment designs simultaneously when the order should be design the mission first, then design the equipment to meet the mission requirements.

In addition to the time-independent real complexity and time-independent imaginary complexity, there are two types of time-dependent complexity: time-dependent combinatorial complexity and time-dependent periodic complexity.

\subsubsection{Combinatorial complexity}

Time-dependent complexity arises when the system range moves as a function of time. Time-dependent combinatorial complexity can lead to a chaotic situation if the number of combinations continues to explode as a function of time or if the underlying physical phenomenon continues to move the system range away from the design range (e.g., wear of sliding surfaces under load).

Time-dependent combinatorial complexity arises because in many situations, the effect of future events on the system cannot be predicted a priori. Many of these problems are combinatorial problems that can grow more complicated indefinitely as a function of time because the future events depend on the decisions made in the past, but in an unpredictable way. In some cases, this unpredictability is due to a violation of the Independence Axiom.

An example of a combinatorial complexity is the airlines' flight schedule in bad weather. Suppose that we have a snowstorm around the Detroit area so that airplanes cannot land and take off. Then the airplanes for Boston cannot take off from Detroit. As time goes on, the flights from Boston to other cities will be disrupted since there will not be enough airplanes to dispatch according to the original schedule. Therefore, the airlines will not be able to satisfy their FRs of sending airplanes on schedule. The situation is going to get worse as time passes and the snowstorm continues. This is an example of time-dependent combinatorial complexity.

It is likely that when the collaboration and negotiation leads into a form of combinatorial complexity, they will lead to more chaotic situation and eventually fail. In this case, an attempt should be made to change this combinatorial complexity problem into a periodic complexity system.

If a system with combinatorial complexity continues to operate for a long time, for instance because the parties involved cannot not collaborate or negotiate (e.g., bankruptcy of a few major US airlines), the system will degenerate into a chaotic state. 


\subsubsection{Periodic complexity}

A system with time-dependent periodic complexity may have an uncertainty only within a period. This type of time-dependent complexity is defined as time-dependent periodic complexity.

Consider again the problem of scheduling airline flights. Although airlines develop their flight schedules, there exist uncertainties in actual flight departures and arrivals because of unexpected events, such as bad weather. The delayed departure or arrival of one airplane will affect many of the subsequent connecting flights and arrival times. However, since the airline schedule is periodic each day, all of the uncertainties introduced during the course of a day terminate at the end of a 24-hour cycle if the weather clears up and aircraft can be relocated during the night when there are not too many flights. Hence, the combinatorial complexity does not extend to the following day, since each day the schedule starts all over again. In a system with a periodic complexity, uncertainties created during the prior period are irrelevant, although during a given period there may be uncertainties.

One of the important implications of the functional periodicity is that when there are many FRs that must be satisfied and if the system has a functional periodicity, the new period must begin after all the FRs are fully satisfied in a given cycle.

To reduce complexity of a system, we may transform a system with a time-dependent combinatorial complexity to a system with time-dependent periodic complexity. The idea is to reinitialise the FRs of the system on a periodic basis, i.e., replace combinatorial complexity with periodic complexity. The period is defined as a functional period. There are many different kinds of functional periodicity (Suh, 2005).

A team collaborating on a project may accrue important beneficial effects if a combinatorial situation can be transformed into a periodic complexity by introducing functional periodicity. A simple example is the introduction of 'time-off' in intense collaboration and negotiation situations.

\subsection{Functional periodicity in collaboration and negotiation}

In designing systems, functional periodicity should be introduced into a system to insure that system that performs a desired set of functions indefinitely. This will make the system stable throughout its lifetime (Suh, 2005). The first step in introducing a functional periodicity is to create uncoupled or decoupled systems by identifying a repeating set of FRs. The system must be reinitialised at the beginning of each period.

To understand the implication of functional periodicity on collaboration, consider the simple case of two parties collaborating on an engineering project. If they find themselves in a disagreement and thus cannot collaborate, they should stop their work and go back and review the highest-level FRs. If their disagreement is not over the original goals of the project represented by the highest-level FRs, they should follow the FR/DP decomposition tree established by their prior collaboration until they identify the source of disagreement. It may turn out that an intermediate-level FR and DP are the source of current conflict, in which case the design should be modified. In other words, the system should be re-initialised to satisfy the highest-level FRs.

The fact that the collaborators are pleased with their collaboration is not a proof that they are doing the right thing. There is also the possibility that both of them are doing a wrong thing and yet are happy. To prevent this situation, a functional periodicity for 
collaboration should be introduced and force the collaborators to re-initialise their original sets of FRs and DPs. This process may reveal the mistakes they have made when they discover inconsistencies in their reasoning, and the decisions they have made.

\section{Issue of local optimum vs. global optimum}

In designing a system, individual designers and engineers may develop a solution that is perceived to be the best for their part of the project. However, there is no guarantee that the solution is the best solution for the entire system. One of the major purposes of collaboration and negotiation is to be sure that the local solutions are consistent with the overall system solution. The axiomatic design framework provides a framework that enables the checking of the local solutions in a global systems context. This is done by making sure that the decomposition is done consistently from a level of hierarchy to the next level of hierarchy, there is no coupling of FRs by means of the design matrix, and by checking the information content.

\section{Case study}

Saab established their corporate goals, strategies, and plans based on the axiomatic design framework, which was used to guide the work and collaboration among its employees (Nordlund, 2006). Nordlund's description of Saab's planning sent to a senior manager of a global US company by e-mail is as follows:

... I have been involved in several planning and strategising sessions where we used the method and axioms (primarily the independence axiom) to develop our plans and strategies. In all these sessions it is safe to say that everyone agreed that the result was much better than it would have been had we not followed this approach. The main reasons for the significantly better result were:

- Zig-Zagging. A clear process, everyone knows what we are doing, and what will happen next.

- Domains. A clear framework to sort information. Information exists at several different abstraction levels and domains simultaneously. The domains provided us with a framework to sort through ideas, analyse old plans, and information.

- Independence axiom. Clear decision making criteria that forces a very pragmatic question at every step: in what sequence shall the different parts of the strategy/plan/action be implemented, and are we sure that there are no conflicting parts of the plan.

- Documentation. Very concise documentation that can serve as an index into underlying documents where more details can be captured. This documentation is also useful a long time after the actual planning session(s).

Saab Service Partner's Business plan 1994-1999 developed Saab's strategic plan, which was used internally and externally. 
M1 Long term support Saab Aircraft and Defense with our expertise, our products and services

M11 Satisfied Existing Customers

M111 Competitive pricing

M112 Functionally customised products

M113 Satisfy customer expectations on quality

M1131 Satisfy customer expectations on product quality

M1132 Satisfy customer expectations on service quality

M1133 Satisfy customer expectations on expertise and personal quality

M12 Increase Market share

M121 Sell existing products to new customers

M122 Sell new products to existing customers

M13 Satisfy Owners

M131 Reduce cost per delivery

M132 Reduce restricted equity (equipment, acct payable, WIP)

The strategies (I restrict the translation to the top level from here on)

S1 Offer Customised and competitive products and services

S11 Prioritise meeting every customer expectation

S111 Monitor market pricing

S112 Study the customers' business, continuous dialog about needs and expectations

S113 Implement processes to meet customer needs

S1131 Systematic development process according to ISO 9000

S1132 Systematic processes according to ISO 9004-2

S1133 Continuously invest in increasing our employees' morale, competence, loyalty, and empowerment

The activities

A1 Continuous Improvement in close cooperation with our customer

A11 Close cooperation with our customers

A111 Analyze markets, competitors, pricing principles

A112 Focus groups (with customers), working groups and networking

A113 Document and develop processes and procedures

A1131

We had a lot of discussions on whether S1133 was a goal or a strategy. It was probably the most interesting part of this whole session, which really made people see the benefit of using axiomatic design. It forced us to really understand the difference of what-how as applied to a company. To keep employees happy is not a goal of a company, however, in successful companies it is an important strategy. In this company's case it was an important strategy to keep their external customers happy. 
In addition to this business plan, we also have a public affirmative action plan that I can share (it is also in Swedish). This was the corporate plan for all of Saab-Scania. This document is in the form of a small pamphlet and was distributed widely in the company.

In closing, some general remarks: Since leaving my MIT fulltime position, I have held senior positions in industry. First as director of technology strategy and acquisition at Saab Corporate, and for the last five years as vice president of engineering at Saab Rosemount Tank Radar AB. My current company was sold from Saab, and is now part of Emerson Electric Corp since five years. I continue using Axiomatic approach when addressing any complex situation with a lot of information and many issues to keep track of. I know of no better way of dealing with complexity.

I comfortably recommend anyone to apply it to planning (I have used it for business planning, technology, market plans, etc.). However, in the first cases it is critical to have a facilitator to run the planning sessions. This is necessary to ensure success. Typically, senior management has little patience for unsuccessful sessions as their time is at a premium (I know from personal experience).

\section{Human elements of collaboration and negotiation}

The preceding discussion of negotiation and collaboration was based on scientific and logical aspects of decision-making when the system hierarchy consists many FRs and DPs at many levels. However, collaboration and negotiation involves real human beings with emotion and self-interest, which introduces another dimension of complication to collaboration and negotiation. When humans interact, the social dynamics will change individuals' perspectives toward a problem, hence lead to different decisions. In the case of a single-person project, choosing FRs and DPs will be purely based on their goodness in delivering the ultimate customer's needs. In a collaborating environment involving people, other issues such as job security, workload, liability, individual ego, etc. are likely to play a significant role. These secondary issues may become a dominating factor in collaboration unless they are properly managed (Lee, 2007). To overcome these difficulties, the following rules have been suggested (Fisher et al., 1991):

- $\quad$ separate the people from the problem (e.g., disentangle and defuse emotional problems)

- focus on interests, not positions (e.g., people often state their position rather than true underlying interests)

- $\quad$ insist on objective criteria

- $\quad$ invent options for mutual gain (e.g. increase the size of the pie before negotiating).

\section{Rules for collaboration and negotiation in design}

The foregoing discussion of axiomatic design and complexity theory yields the following heuristic rules that may help the project and the collaborating participants: ${ }^{2}$ 
Rule 1: All participants must agree, before proceeding, on the customer who must be satisfied, and the attributes that the customer is looking for.

Rule 2: All participants must agree on the highest-level FRs defined for the project through collaboration.

Rule 3: In establishing the FRs, make sure that the FRs are independent of each other by checking whether they are stating the same thing using different units or words. FRs are independent of each other by definition.

Rule 4: The Independence of FRs is not negotiable.

Rule 5: In selecting DPs to satisfy the FRs, select the DPs that maintain the independence of FRs by checking the DPS (acceptable Design Matrix must be diagonal or triangular).

Rule 6 If possible, check the system range of the FRs chosen against the design range to choose the best DP. Sometimes, it is difficult to know the system range when the details of the design have not been developed through decomposition.

Rule 7: In selecting PVs to enable the creation of DPs, select PVs that maintain the independence of DPs. PVs are manufacturing processes, but in organisational design, PVs are resources.

Rule 8: Check if the chosen PV is likely to satisfy the DP by checking the required tolerance of DPs and the tolerance of a DP that can be created by the chosen PV.

Rule 9: Complexity should be minimised or reduced.

Rule 10: Evaluate the collaborative effort to determine if it has the characteristics of combinatorial complexity, which is indicated by continuing deterioration of the output with time.

Rule 11: Introduce a functional periodicity in collaboration and negotiation by forcing all the participants to review the established FRs at all levels, and reinitialise the project for the following period as a means of preventing the collaboration from becoming a process with combinatorial complexity.

Rule 12: Check the design matrix periodically to be sure that the collaborative project is not working on an imaginary complexity problem.

Rule 13: If the system has real complexity, redesign the system starting from the highest-level FRs.

Rule 14: The impact of changes must be assessed and all affected FRs and DPs must be modified.

Rule 15: All participants must honour decisions made at higher levels. If the lower-level decisions indicate that the higher-level decisions had a flaw, then the higher-level decisions should be reviewed and corrected.

Rule 16: Negotiated decisions must be self-consistent throughout the hierarchy.

Rule 17: Constraints must be tracked and should not be violated. 


\section{Other suggestions for collaboration process}

The following suggestions may be helpful for all the participants to remember.

- the 'design architect' should be the only one who can make the changes of the FR/DP system hierarchy and the design matrix so as to eliminate the possibility of introducing changes to the system architecture without approval

- comments should be inserted to provide rationale for the decisions made

- $\quad$ use common software (e.g., Acclaro)

- distributed decision systems must be employed for large projects after agreeing on the FRs of each branch

- $\quad$ basic understanding of the design theory should be required.

\section{Conclusions}

The quality and productivity of technological and industrial endeavors depends on human interactions that lead to decisions. Collaboration and negotiation are important elements of group-decision making. The current ad hoc process of collaboration and negotiation should benefit when it is based on scientific principles and approaches. Specific conclusions are as follows:

- collaboration and negotiation in developing engineering systems are necessary in executing large projects

- the field of collaboration and negotiation in engineering needs to establish basic principles that can make the field more rational and efficient

- a set of rules is proposed that can be used by all participants in engineering system design to promote further discussion.

\section{Acknowledgements}

The author gratefully acknowledges the helpful suggestions and comments made by Professor Christopher A. Brown, Dr. Taesik Lee, Professor Stephen Lu, and Dr. Nannaji Saka, and the editorial assistance of Mr. Tony Pulsone. This research was funded by the Park Center for Complex Systems at MIT.

\section{References}

Arrow, K.J. (1951) (2nd ed. 1963) Social Choice and Individual Values, Wiley, New York.

Branscomb, L.M. and Auerswald, P.E. (2001) Taking Technical Risks: How Innovators, Executives, and Investors Manage High-Tech Risks, The MIT Press, Cambridge, MA, USA

Fisher, R., Ury, W. and Patton, B. (1991) Getting to Yes: Negotiating Without Giving In, Penguin, New York, NY, USA. 
Harsanyi, J.C. (1955) 'Cardinal welfare, individualistic ethics, and interpersonal comparisons of utility', Journal of Political Economy, Vol. 63, pp.309-321.

Keeney, R.L. (1976) 'Group preference axiomatisation with cardinal utility', Management Science, Vol. 23, pp.140-143.

Lee, T. (2007) Personal Communication, MIT, Cambridge, MA, USA.

Lu, S. (2006a) 'A socio-technical foundation for collaborative engineering design', CIRP Annuals, Vol. 56, No. 2, pp.605-634.

Lu, S. (2006b) Personal Communication.

Nordlund, M. (2006) E-mail Message, 11 January.

Sohlenius, G. (2005) 'Systematic nature of engineering to obtain welfare through profitable fulfillment of customer needs', ICAM 2005 International Conference on Agility, Helsinki, July, pp.27, 28.

Suh, N.P. (1990) The Principles of Design, Oxford University Press, NY.

Suh, N.P. (2001) Axiomatic Design: Advances and Applications, Oxford University Press, NY.

Suh, N.P. (2005) Complexity: Theory and Applications, Oxford University Press, NY.

\section{Notes}

${ }^{1}$ Not all innovations require collaboration and negotiation, especially when it is done serially. Many inventive ideas are often conceived in the head of one person, who has the ability to identify the customer needs, define the Functional Requirements (FRs) of a new product, and invent a new product that can satisfy the FRs. Once the viability of the invention is proven, it is turned over to other groups sequentially for development, manufacturing, marketing, and so on, with limited collaboration and negotiation. In many corporations this serial process is not well accepted when the product is mass-produced and if the investment required is large.

${ }^{2}$ These rules have not yet been tested and verified for correctness. 